\title{
Evolution of quasistationary electron spectrum in open spherical quantum dot
}

\author{
M.V.Tkach, Ju.O.Seti, O.M.Voitsekhivska \\ Fedkovych Chernivtsi National University, 2, Kotsyubinskoho St., Chernivtsi, 58012, Ukraine*
}

Received February 27, 2008

\begin{abstract}
The evolution of electron quasistationary spectrum in open spherical quantum dot is under study within the effective mass and rectangular potential model. Within the framework of the S-matrix model the exact solution of Schrödinger equation is obtained in general analytical form. It is shown, for the first time, that the generalized resonance energies and widths introduced as the parameters defining the probability distribution function (over the energy or quasi momentum) of electron location in quantum dot, adequately characterize the evolution of its quasistationary states (contrary to the S-matrix poles) in the whole range of barrier thickness: from zero (free states) up to the infinity (stationary bound states are under the barrier and virtual and free states are above it).
\end{abstract}

Key words: spherical quantum dot, quasistationary state

PACS: 71.15.Dx, 73.21.La, 73.22.f, 73.90.+f

\section{Introduction}

The open nanosystems, in general, and quantum dots, in particular, attract more attention of researches [1-3]. Recently, the essentially increasing interest to them has been caused by the important role they play not only in physics but in microbiology and medicine [4-6] as well.

Naturally, the study of physical phenomena in open nanosystems begins from the development of the theory of quasiparticle spectra and their interaction with quantized and classic fields. There is still no straight and consistent theory of interaction in nanosystems due to the principal mathematical difficulties arising while attempting to use the quantum field methods for these systems. Moreover, the complications arise even while calculating the quasiparticle quasistationary spectra (e.g., exciton as an interacting electron and hole).

The investigations of the electron or hole quasistationary spectra in quantum dots (QD) and wires have been performed in [7-12] solving the Schrödinger equation within the S-matrix method. A comparatively simple model of effective masses and rectangular potential barriers for an electron made it possible to obtain the exact analytical expressions for the S-matrix but the calculation of the resonance energies and widths of quasistationary states could be performed only for nanosystems with rather "powerful" barriers, when these magnitudes were defined by the S-matrix poles in a complex plane.

According to the scattering theory [13], for a small "power" of potential barrier, the S-matrix poles already do not define the parameters of quasistationary spectra. Such a situation frequently happens while researching quasiparticle spectra in open QDs when the barrier thickness is rather small, i.e., not bigger than several monoshells [3]. Thus, there is a problem of introducing such characteristics of quasiparticle spectrum in open spherical quantum dot (OSQD), which would be unique and just for the whole range of barrier thicknesses: from zero (free states) to infinity (stationary states).

In this paper, we develop a theory of electron quasistationary spectrum in OSQD with arbitrary barrier thickness. Herein, it is shown, for the first time, that the universal spectral parameter describing the evolution of generalized resonance energies and widths of quasistationary states

*E-mail: theorphys@chnu.cv.ua 
from free to bound stationary is the probability distribution function (over quasimomentum or energy) of electron location in QD. It is shown that the introduced conception of generalized resonance energies and widths of quasistationary bands is equivalent to the resonance energies and bandwidths determined by the S-matrix poles at rather big barrier thicknesses and, according to the physical considerations, transfer into the respective spectral characteristics of free states at limiting small barrier thicknesses.

\section{Hamiltonian, S-matrix and probability distribution function of electron lo- cation within OSQD}

While developing the theory of electron quasistationary spectrum in OSQD (figure 1) it is assumed that the radius of the core-well $\left(r_{0}\right)$ and the barrier thickness $(\Delta)$ are fixed. Within the framework of the effective mass approximation $(m)$ and rectangular potential barrier model, the electron Hamiltonian is as follows:

$$
H=-\frac{\hbar^{2}}{2 m} \overrightarrow{\nabla^{2}}+U(r),
$$

where the potential $U(r)$ in the spherical coordinate system with the reference in the center is written as follows:

$$
U(r)= \begin{cases}0, & 0 \leqslant r \leqslant r_{0}, \quad r_{0}+\Delta \leqslant r<\infty \\ U, & r_{0} \leqslant r \leqslant r_{1}=r_{0}+\Delta .\end{cases}
$$

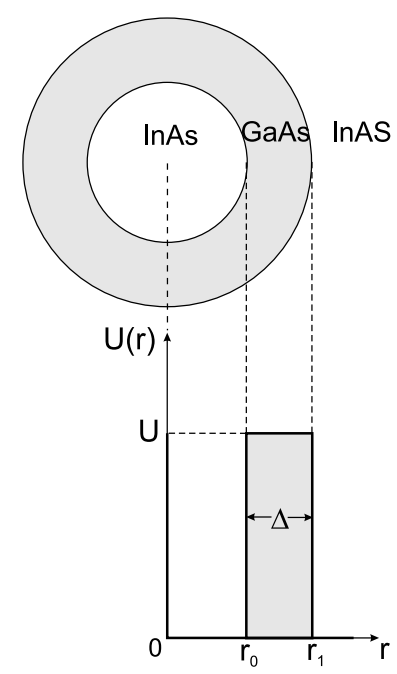

Figure 1. Geometric and potential energy schemes of nanosystem.

Taking into account the spherical symmetry, the solutions of Schrödinger equation with Hamiltonian (1) are the wave functions

$$
\Psi_{\ell m}(r, \theta, \varphi)=R_{\ell}(r) Y_{\ell m}(\theta, \varphi)
$$

where $Y_{\ell m}(\theta, \varphi)$ are the spherical functions $(\ell=0,1,2, \ldots ; m=0, \pm 1, \pm 2, \ldots)$. It is convenient to take the radial functions in under-barrier energy region $(E<U)$ as the linear combinations of Hankel functions

$$
R_{\ell k}= \begin{cases}R_{\ell}^{(0)}(k r)=a_{\ell}^{(0)}\left[h_{\ell}^{-}(k r)+h_{\ell}^{+}(k r)\right], & 0 \leqslant r \leqslant r_{0}, \\ R_{\ell}^{(1)}(k r)=a_{\ell}^{(1)}\left[h_{\ell}^{-}(i \chi r)+S_{\ell}^{(1)} h_{\ell}^{+}(i \chi r)\right], & r_{0} \leqslant r \leqslant r_{1}=r_{0}+\Delta, \\ R_{\ell}^{(2)}(k r)=a_{\ell}^{(2)}\left[h_{\ell}^{-}(k r)+S_{\ell} h_{\ell}^{+}(k r)\right], & r_{0}+\Delta \leqslant r<\infty\end{cases}
$$


where

$$
k=\frac{\sqrt{2 m E}}{\hbar}, \quad \chi=\sqrt{k_{0}^{2}-k^{2}}, \quad k_{0}=\frac{\sqrt{2 m U}}{\hbar} .
$$

The radial wave function and density of probability current continuity conditions at the both nanosystem interfaces

$$
\begin{array}{ll}
R_{\ell}^{(0)}\left(k r_{0}\right)=R_{\ell}^{(1)}\left(i \chi r_{0}\right), & \left.\frac{\mathrm{d} R_{\ell}^{(0)}(k r)}{\mathrm{d} r}\right|_{r=r_{0}}=\left.\frac{\mathrm{d} R_{\ell}^{(1)}(i \chi r)}{\mathrm{d} r}\right|_{r=r_{0}}, \\
R_{\ell}^{(1)}\left(i \chi r_{1}\right)=R_{\ell}^{(2)}\left(k r_{1}\right), & \left.\frac{\mathrm{d} R_{\ell}^{(1)}(i \chi r)}{\mathrm{d} r}\right|_{r=r_{1}}=\left.\frac{\mathrm{d} R_{\ell}^{(2)}(k r)}{\mathrm{d} r}\right|_{r=r_{1}},
\end{array}
$$

and the normalizing condition

$$
\int_{0}^{\infty} R_{\ell k^{\prime}}^{*}(r) R_{\ell k}(r) r^{2} \mathrm{~d} r=\delta\left(k-k^{\prime}\right)
$$

determine all the coefficients $a_{\ell}^{(0)}, a_{\ell}^{(1)}, a_{\ell}^{(2)}, S_{\ell}^{(1)}$ and $S_{\ell}$-matrix.

Finally, the analytical calculations bring us to the following results

$$
\begin{aligned}
& a_{\ell}^{(0)}=\frac{h_{\ell}^{-}\left(i \chi r_{0}\right)+S_{\ell}^{(1)} h_{\ell}^{+}\left(\mathrm{i} \chi r_{0}\right)}{h_{\ell}^{-}\left(k r_{0}\right)+h_{\ell}^{+}\left(k r_{0}\right)} a_{\ell}^{(1)}, \\
& a_{\ell}^{(1)}=\frac{h_{\ell}^{-}\left(k r_{1}\right)+S_{\ell} h_{\ell}^{+}\left(k r_{1}\right)}{h_{\ell}^{-}\left(i \chi r_{1}\right)+h_{\ell}^{+}\left(i \chi r_{1}\right)} a_{\ell}^{(2)}, \quad a_{\ell}^{(2)}=-\frac{\mathrm{i} k}{\sqrt{2 \pi}}, \\
& S_{\ell}^{(1)}=-\frac{m_{1} k^{2} h_{\ell}^{-}\left(\mathrm{i} \chi r_{0}\right) j_{\ell}^{\prime}\left(k r_{0}\right)+m_{0} \chi^{2} j_{\ell}\left(k r_{0}\right) h_{\ell}^{-^{\prime}}\left(\mathrm{i} \chi r_{0}\right)}{m_{1} k^{2} h_{\ell}^{+}\left(\mathrm{i} \chi r_{0}\right) j_{\ell}^{\prime}\left(k r_{0}\right)+m_{0} \chi^{2} j_{\ell}\left(k r_{0}\right) h_{\ell}^{+{ }^{\prime}}\left(\mathrm{i} \chi r_{0}\right)},
\end{aligned}
$$

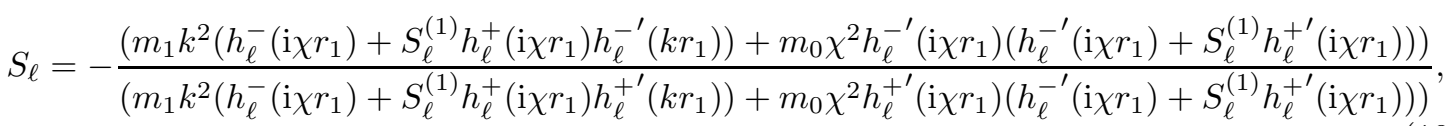

where

$$
\begin{aligned}
h_{\ell}^{ \pm \prime}\left(\mathrm{i} \chi r_{0,1}\right) & =\left.\frac{\partial h_{\ell}^{ \pm}(x)}{\partial x}\right|_{x=\mathrm{i} \chi r_{0,1}}, \quad j_{\ell}^{\prime}\left(k r_{0}\right)=\left.\frac{\partial j_{\ell}(x)}{\partial x}\right|_{x=k r_{0}}, \\
j_{\ell}(x) & =\frac{1}{2}\left(h_{\ell}^{-}(x)+h_{\ell}^{+}(x)\right) .
\end{aligned}
$$

The probability distribution functions $W_{\ell}(k)$ and $W_{\ell}(E)$ (over quasimomentum $k$ or energy $E$ ) of electron location within spherical OSQD can be found from the following formulas

$$
W_{\ell}(E)=\frac{1}{r_{1}} \int_{0}^{r_{1}}\left|R_{\ell E}(r)\right|^{2} r^{2} \mathrm{~d} r, \quad W_{\ell}(k)=\frac{1}{r_{1}} \int_{0}^{r_{1}}\left|R_{\ell}(k r)\right|^{2} r^{2} \mathrm{~d} r .
$$

For the two close magnitudes of energies $\left(E, E_{1}\right)$, using the function $X_{\ell E}(r)=R_{\ell E}(r) / r$ and from the Schrödinger equation, in a limiting case one can obtain

$$
W_{\ell}(E)=\frac{1}{r_{1}} \int_{0}^{r_{1}} \mathrm{X}_{E}^{2} \mathrm{~d} r=\left.\frac{\hbar^{2}}{2 m} \lim _{E_{1} \rightarrow E} \frac{X_{E_{1}} \mathrm{X}_{E}^{\prime}-\mathrm{X}_{E_{1}}^{\prime} \mathrm{X}_{E}}{E_{1}-E}\right|_{r=r_{1}} .
$$

Since at $r \geqslant r_{1}, U(r)=0$, then, according to the theory [13], for this range of radii it is written

$$
X_{\ell E}^{2}(r)=\sqrt{\frac{2}{\pi}} \sin \left(k r+\delta_{\ell}\right),
$$


where the phase $\left(\delta_{\ell}\right)$ is connected with the $S_{\ell}$-matrix by the relation

$$
S_{\ell}(k)=\mathrm{e}^{2 \mathrm{i} \delta_{\ell}(k)} .
$$

Inserting (14) into (13), we obtain the probability distribution function, as certain generalization of Luders formula, [13]

$$
W_{\ell}(k)=\frac{1}{\pi r_{1}}\left[r_{1}+\frac{\mathrm{d} \delta_{\ell}(k)}{\mathrm{d} k}-\frac{1}{2 k} \sin 2\left(k r_{1}+\delta_{\ell}(k)\right)\right] .
$$

On the one hand, taking into account the $S_{\ell}(k)$-matrix, (10) and its relationship to the phase $\delta_{\ell}(k),(15)$ and, on the other hand, expressing the $S_{\ell}$-matrix (for the system under research) through the real $Z_{\ell}(k)$-matrix

$$
S_{\ell}(k)=\mathrm{e}^{-2 \mathrm{i} k r_{1}} \frac{1+\mathrm{i} Z_{\ell}(k)}{1-\mathrm{i} Z_{\ell}(k)},
$$

where

$$
\begin{gathered}
Z_{\ell}(k)=(-1)^{\ell}\left[\frac{F_{\ell}(k) \operatorname{Im} f_{\ell}\left(\mathrm{i} k r_{1}\right)-\operatorname{Re} f_{\ell+1}\left(\mathrm{i} k r_{1}\right)}{F_{\ell}(k) \operatorname{Im} f_{\ell}\left(\mathrm{i} k r_{1}\right)+\operatorname{Re} f_{\ell+1}\left(\mathrm{i} k r_{1}\right)}\right]^{ \pm 1}, \quad(", ", \ell=2,4,6, \ldots), \\
F_{\ell}(k)=\frac{\chi}{k} \frac{\mathrm{e}^{-2 \chi \Delta} f_{\ell+1}\left(\chi r_{1}\right) f_{\ell}\left(-\chi r_{0}\right) \Phi_{\ell}\left(k r_{0}\right)+f_{\ell+1}\left(-\chi r_{1}\right) f_{\ell}\left(\chi r_{0}\right)}{\mathrm{e}^{-2 \chi \Delta} f_{\ell}\left(\chi r_{1}\right) f_{\ell}\left(-\chi r_{0}\right) \Phi_{\ell}\left(k r_{0}\right)-f_{\ell}\left(-\chi r_{1}\right) f_{\ell}\left(\chi r_{0}\right)}, \\
\Phi_{\ell}\left(k r_{0}\right)=\frac{f_{\ell}\left(\chi r_{0}\right)}{f_{\ell}\left(-\chi r_{0}\right)} \frac{k \varsigma_{\ell+1}\left(k r_{0}\right) f_{\ell}\left(-\chi r_{0}\right)+\chi f_{\ell+1}\left(-\chi r_{0}\right) \varsigma_{\ell}\left(k r_{0}\right)}{k \varsigma_{\ell+1}\left(k r_{0}\right) f_{\ell}\left(\chi r_{0}\right)-\chi f_{\ell+1}\left(\chi r_{0}\right) \varsigma_{\ell}\left(k r_{0}\right)} \\
\varsigma_{\ell}\left(k r_{0}\right)=\sum_{s=0}^{\ell} \frac{(\ell+s) !\left[\operatorname{Re}\left(\mathrm{i}^{s-\ell-1}\right) \cos k r_{0}+\operatorname{Im}\left(\mathrm{i}^{s-\ell+1}\right) \sin k r_{0}\right]}{s !(\ell-s) !\left(2 k r_{0}\right)^{s}} \\
f_{\ell}(x)=\sum_{s=0}^{\ell} \frac{(\ell+s) !}{s !(\ell-s) !(2 x)^{s}}
\end{gathered}
$$

one can obtain from (16) a simple and exact analytical expression for the probability distribution function $W_{\ell}(k)$ of electron in the state with orbital number $\ell$ in OSQD

$$
W_{\ell}(k)=\frac{k \frac{\mathrm{d}\left(Z_{\ell}(k) / k\right)}{\mathrm{d} k}}{\pi r_{1}\left(1+Z_{\ell}^{2}(k)\right)} .
$$

It is found that just this function (or $W_{\ell}(E)$ ) contains the total information about all free and virtual quasistationary and stationary states of electron at arbitrary barrier thicknesses (varying form zero to infinity), rather than the $S_{\ell}(k)$-matrix poles. These functions are valid even for $k \geqslant k_{0}$ at the condition $\chi \rightarrow \mathrm{i} \chi$.

\section{Evolution of electron quasistationary states from free to bound when the barrier thickness varies from zero to infinity}

The numerical calculations of $W_{\ell}(k)$ and $W_{\ell}(E)$ probability distribution functions of electron location in OSQD (InAs/GaAs/InAs) with physical parameters: $m_{\mathrm{e}}=0.0445 m_{0}, U=535 \mathrm{meV}$, $a_{\mathrm{B}}=0.529 \AA, a_{0}=a_{\mathrm{InAs}}=6.058 \AA, a_{1}=a_{\mathrm{GaAs}}=5.653 \AA$ (figure 1 ) are performed according to the theory developed earlier. In order to analyze the evolution of generalized resonance energies and widths of electron quasistationary states as functions of OSQD geometric sizes (radius $r_{0}$ and barrier thickness $\Delta$ ) one has to study their main properties and introduce their definitions, valid at the whole range of barrier thicknesses and totally coinciding with the resonance energies and widths determined by $S$-matrix poles in complex plane at $\chi \Delta \gg 1$. 

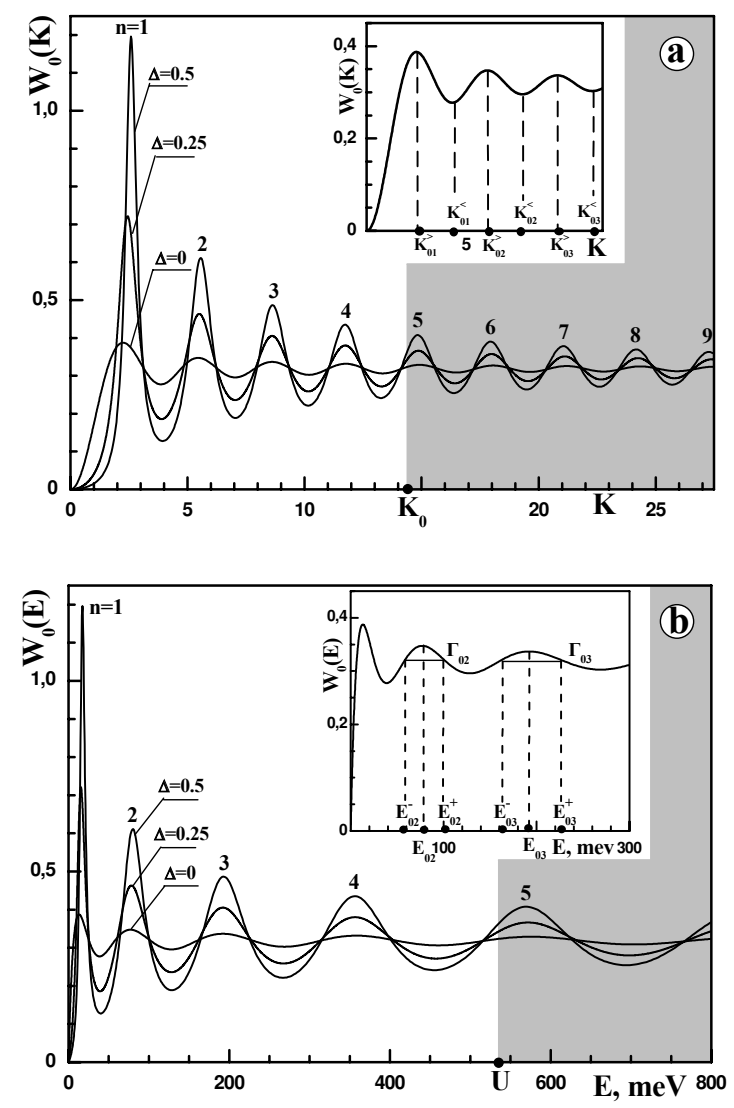

Figure 2. Dependences of $W_{0}(K)$ (a) and $W_{0}(E)$ (b) for very small barrier thicknesses $(0 \leqslant$ $\left.\Delta \ll r_{0}\right) . K=k r_{0}, r_{0}=30 a_{0}$.

First of all we are going to study $W_{0}(k), W_{0}(E)$ functions, watching their evolution in spherically-symmetric states $(\ell=0)$, figures 2,3 . The analysis proves that their properties essentially differ for the three different intervals of barrier thicknesses: small $\left(0 \leqslant \Delta \ll r_{0}\right)$, relative $\left(\Delta \approx r_{0}\right)$ and large $\left(\Delta \gg r_{0}\right)$.

For the small thicknesses, one can see from figure $2 \mathrm{a}, \mathrm{b}$ and analytical expression

$$
W_{0}(\Delta=0, k)=\frac{1}{\pi}\left(1-j_{0}(k r)\right)=\frac{1}{\pi}\left(1-\frac{\sin 2 k r}{2 k r}\right)
$$

that, even when there is no barrier $(\Delta=0)$, the probability distribution functions of quasiparticle location in the sphere of certain radius $(r)$, depending on $k$ or $E$, perform quasiperiodical oscillations relatively to the average value

$$
\bar{W}_{0}=\lim _{A \rightarrow \infty} \frac{1}{A} \int_{0}^{\infty} W_{0}(k) \mathrm{d} k=\frac{1}{\pi}
$$

consistently taking minimum (sign " $<$ ") and maximum (sign " $>$ ") magnitudes

$$
W_{n}^{<}=\frac{2}{\pi} \sin ^{2} k_{n}^{<} r, \quad W_{n}^{>}=\frac{2}{\pi} \sin ^{2} k_{n}^{>} r \quad(n=0,1,2, \ldots, \infty),
$$

where

$$
k_{n}^{<}=\frac{x_{2 n}}{2 r}, \quad k_{n}^{>}=\frac{x_{2 n+1}}{2 r}
$$


with $x_{2 n}$ and $x_{2 n+1}$ being the even and odd roots of the equation

$$
W^{\prime}(x)=0, \quad \text { or } \quad j_{1}(x)=0, \quad \text { or } \quad x \cot x=1 .
$$

Finally, at $\Delta=0, W_{0}(k), W_{0}(E)$ functions describe the spherically-symmetric free states of the quasiparticle in $k$ - or $E$-space.

When the potential barrier $U$ of a small thickness $\Delta$ appears at the distance $r_{0}$ from the center of the system, the both functions change, as one can see in figure $2 \mathrm{a}$, b. It is obvious that the increase of $\Delta$ leads to the increase of the amplitude shifts for all peaks. Herein, the locations of the $W_{0}(k)$ and $W_{0}(E)$ maxima in $k$ or $E$ scales change weakly, and the magnitudes of $W_{0}$ maxima themselves increase rapidly while the peaks take the shape of quasi-Lorentz curves. Therefore, it is convenient to introduce two spectral characteristics of each separate peak: generalized resonance energy $\left(E_{0 n}=\right.$ $\left.\frac{\hbar^{2}\left(k_{n}^{>}\right)^{2}}{2 m}\right)$, corresponding to the maxima $W_{0 n}\left(k_{n}^{>}\right)$magnitudes and generalized resonance width $\left(\Gamma_{0 n}=E_{0 n}^{(+)}-E_{0 n}^{(-)}\right)$. The energies $E_{n}^{( \pm)}$are fixed by the typical condition (figure 2)

$$
2 W_{0}(E)=W_{0}\left(k_{n}^{>}\right)+W_{0}\left(k_{n}^{<}\right) .
$$

The magnitudes $E_{0 n}$ and $\Gamma_{0 n}$, introduced at $\Delta=0$, define the generalized resonance energies and widths of the quasiparticle free virtual states. When the barrier appears and its thickness increases, $E_{0 n}(\Delta)$ and $\Gamma_{0 n}(\Delta)$ magnitudes change due to the change of $W_{0}(k), W_{0}(E)$ functions.

From figure $2 \mathrm{a}, \mathrm{b}$ it is clear that for very small thicknesses, the increase of $\Delta$ causes the increase of probability density in the vicinity of generalized resonance energies due to their decrease in the intervals between the resonances. This concerns both the under-barrier (light background in the figure), and the above-barrier (grey background) regions. Physically, it means that at $\Delta$ increasing, the states are gradually transforming from free one at $\Delta=0$ into the quasistationary one with decreasing resonance widths. Herein, the shape of the peaks and their location in under- and above barrier regions (over $k$ and $E$ ) do not differ essentially.
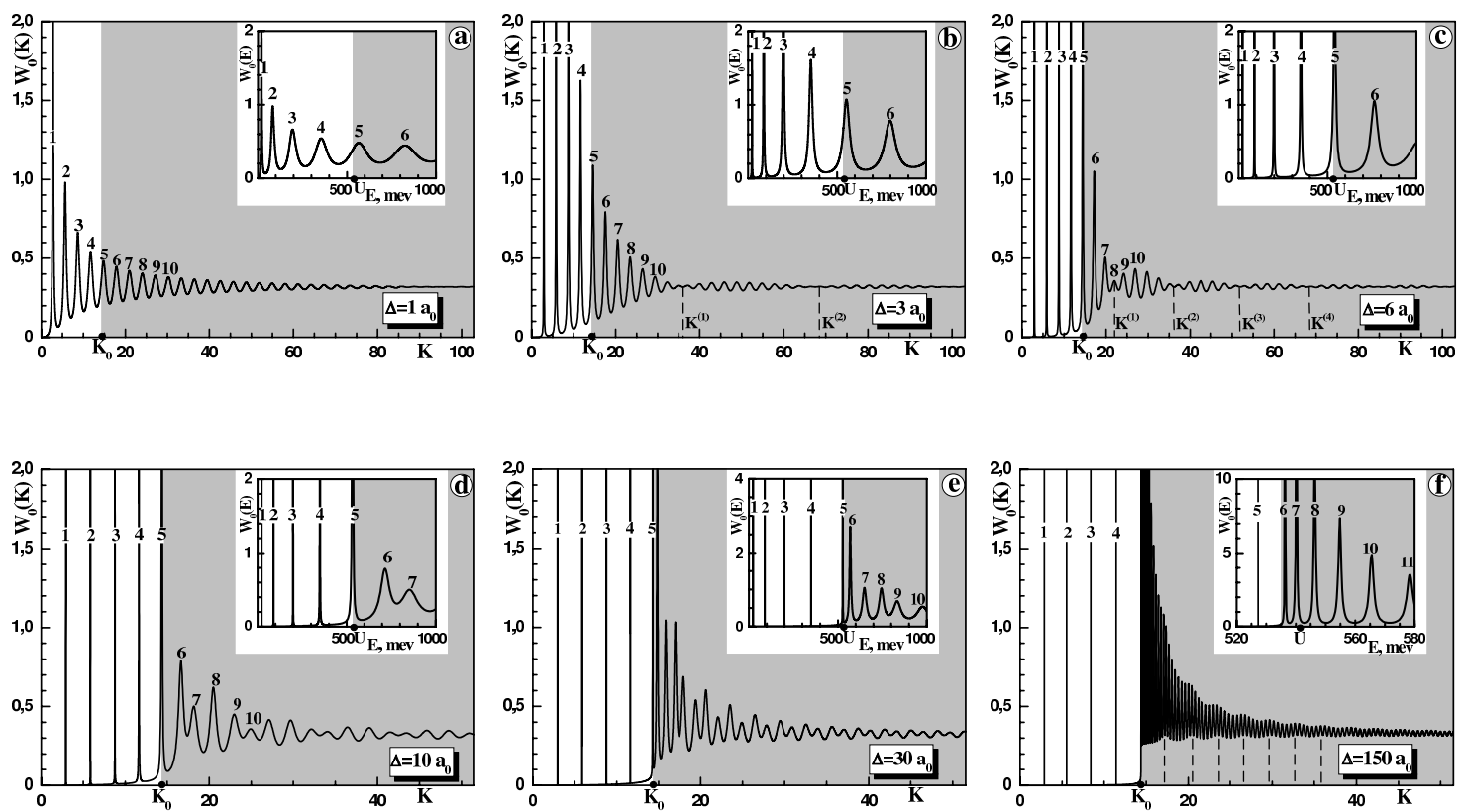

Figure 3. Dependences of $W_{0}(K)$ and $W_{0}(E)$ for small $\left(\Delta<r_{0}, \mathrm{a}, \mathrm{b}, \mathrm{c}\right)$, relative $\left(\Delta \approx r_{0}, \mathrm{~d}, \mathrm{e}\right)$ and large $\left(\Delta \gg r_{0}, \mathrm{f}\right)$ thicknesses. $K=k r_{0}, r_{0}=30 a_{0}$.

A more interesting picture of the spectrum formation is observed for the barrier thicknesses relative to the radius $r_{0}$ (figure $3 \mathrm{a}-\mathrm{d}$ ) and equal or much bigger than the radius $r_{0}$ (figure $3 \mathrm{e}, \mathrm{f}$ ). We are going to analyze the evolution of the spectrum in $k$ - or $E$-space dividing them into underbarrier $\left(k \leqslant k_{0}, E \leqslant U\right)$ and above-barrier $\left(k \geqslant k_{0}, E \geqslant U\right)$ regions. Figure 3 presents the following properties of $W_{0}(k), W_{0}(E)$ functions. 
For the under-barrier energies, all peaks have the shape of quasi-Lorentz curves, characterized by generalized resonance energies $E_{0 n}(\Delta)$ and widths $\Gamma_{0 n}(\Delta)$. The increase of barrier thickness causes a rapid decrease of $\Gamma_{0 n}(\Delta)$ generalized resonance widths of all peaks (their heights increase). Herein, the $E_{0 n}(\Delta)$ generalized resonance energies change weakly. At any fixed thickness, the increase of a peak order number $(n)$ causes an increase of its generalized resonance width $\Gamma_{0 n}$ and the height becomes smaller. As far as figure 3 proves, the tops of the peaks in $k$-scale are located almost equidistantly. Then, the difference between the neighbour resonance energies is: $\Delta E_{0 n}=$ $E_{0 n+1}-E_{0 n}$ in $E$-scale and, thus, the generalized resonance widths $\Gamma_{0 n}$ increase proportionally to $k_{0 n}$ magnitude. We should note that at the thicknesses $\Delta=30 a_{0}, 150 a_{0}$, the widths of under-barrier peaks (in the presented scale) are so small that they have the shape of thin lines ( $\delta$-like peaks) located at such energies which are almost equal to the energies of stationary electron spectrum in the closed $(\Delta \rightarrow \infty)$ spherical QD.

No less interesting is the formation of the above-barrier states spectrum. As one can see in figure 3, in OSQD with $\Delta<r_{0}$ thickness, $W_{0}(k), W_{0}(E)$ functions oscillate in the above-barrier region differently than in the under-barrier region. The pre-barrier region is created when $k_{0}$ increases to $k$. In this region, the height of every next peak becomes smaller till the minimum value at $k=k^{(1)}$. Further, there appears the first packet of states, where the heights of peaks at first grow from $k^{(1)}$ and then decrease up to the minimum value at $k=k^{(2)}$. Then, the second, the third and the next packets of states arise. The pre-barrier region sizes (over $k$ ) and that of all states packets decrease when the barrier thickness increases. Herein, the heights of the first pre-barrier peaks and the highest peaks in every state packet become bigger.

Such an evolution of $W_{0}(k)$ function occurs up to the thickness (in this case $\Delta \sim 10 a_{0}$ ), at which the pre-barrier region and the first states packet contain only two peaks. The further increases of the thickness bring about the "washout" of states packets (figure $3 \mathrm{~d}$ ). At $\Delta \gg r_{0}$, the amplitudes of $W_{0}(k)$ oscillations are smoothly decreasing and for the envelope function of the up and down parts of peaks there is observed a "modulation" of oscillations with maxima located in the vicinity of generalized resonance energies of virtual states (figure 2a).

Contrary to the under-barrier peaks, weakly shifting in $E$-scale at $\Delta$ increasing from zero to infinity, all the above-barrier peaks rapidly shift from their positions in virtual states into low energy under-barrier region creating there a quasicontinuum distribution (figure 3). Further on, it becomes denser transiting into the continuum distribution at $\Delta \rightarrow \infty$.

Mathematically different behavior of $W_{0}(k), W_{0}(E)$ in under- and above-barrier regions is mainly caused by the multiplier $\mathrm{e}^{-2 i \chi \Delta}$, real at $k \leqslant k_{0}$. At $k \leqslant k_{0}$ it transforms into the complex one $\exp ^{-2 \chi \Delta}$, determining the character of oscillations in the above-barrier region (states packets at $\Delta \approx r_{0}$ and "modulated" oscillations $W_{0}(k)$ at $\left.\Delta \gg r_{0}\right)$.

Figure 4 proves that all properties of $W_{0}(k), W_{0}(E)$ functions for spherically symmetric states are similar for the states with $\ell \neq 0$. One has to note that the increase of the orbital quantum number leads to an increase of the generalized resonance energies $E_{\ell n}$ and widths $\Gamma_{\ell n}$.

Finally, the general physical picture of the evolution of electron quasistationary spectrum in OSQD as a function of the potential barrier thickness is as follows: when there is no potential barrier $(\Delta=0)$, the electron freely moves from a certain point which is the center of the sphere with an arbitrary $r_{0}$ radius. The probability of electron location within this sphere is characterized by $W_{\ell}(E)$ or $W_{\ell}(k)$ distribution functions with infinite number of peaks depending on the magnitude of radial quasimomentum $(k)$ or energy $(E)$. Each peak corresponds to a certain electron state and is characterized by resonance energies $\left(E_{\ell n}\right)$, at which the electron has the maximum probability of locating within the sphere with $r_{0}$ radius, and generalized resonance widths $\left(\Gamma_{\ell n}\right)$ defining the life times $\left(\tau_{\ell n}=\hbar \Gamma_{\ell n}^{-1}\right)$ in these states.

When the potential barrier $U$ with arbitrary small thickness $\Delta$ appears in the space where the electron moves at a distance $r_{0}$ from the center, all free states become quasistationary. When $\Delta$ is increasing the peaks of quasistationary states are modifying in such a way that under the barrier their form at first becomes quasi-Lorentz, then Lorentz and finally $\delta$-like (at $\Delta \rightarrow \infty$ ), corresponding to the bound stationary states of the closed SQD. At the increase of the barrier thickness, the above barrier peaks, characterizing the corresponding electron states, present the 

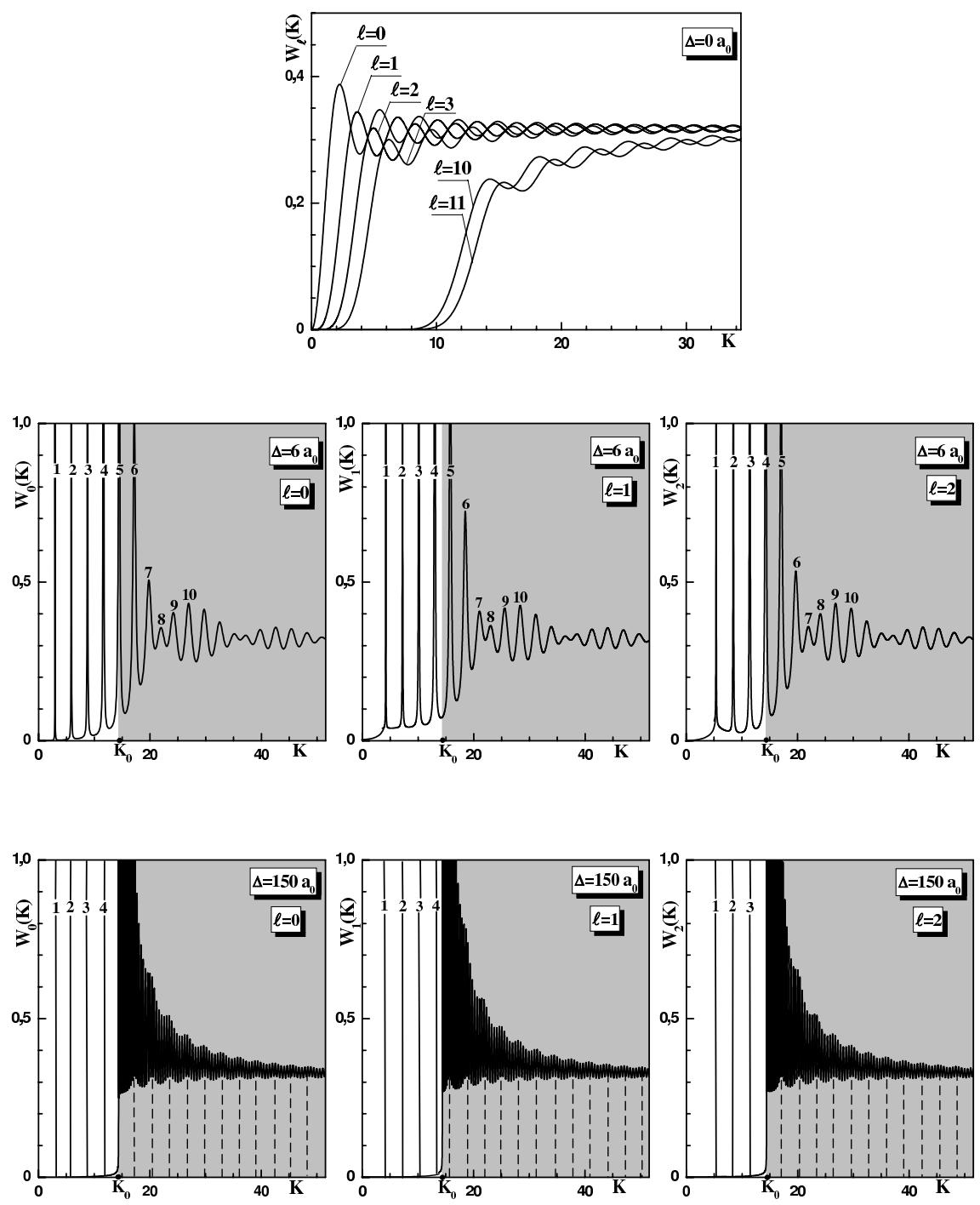

Figure 4. Dependence of $W_{\ell}$ on $K$ for different orbital quantum numbers and barrier thicknesses $\Delta . K=k r_{0}, r_{0}=30 a_{0}$.

already described complicated evolution through the different quasistationary states to the states of continuum spectrum at $\Delta \rightarrow \infty$.

Analysis of $W_{\ell}(k)$ and $W_{\ell}(E)$ probability distribution functions proves that the introduced generalized resonance energies $\left(E_{\ell n}^{W}\right)$ and widths $\left(\Gamma_{\ell n}^{W}\right)$ adequately describe the spectral characteristics of quasistationary electron states at infinite interval of barrier thickness $(0 \leqslant \Delta \leqslant \infty)$. Now, one can be convinced that $E_{\ell n}^{W}$ and $\Gamma_{\ell n}^{W}$ magnitudes at rather large thicknesses coincide well to the respective resonance energies $\left(E_{\ell n}^{S}\right)$ and widths $\left(\Gamma_{\ell n}^{S}\right)$, defined as the poles of $S_{\ell}(k)$-matrix in complex plane. Besides, on the contrary to the latter, contradicting to the Heisenberg principle of indeterminacy at the small thicknesses and, since can not describe the physical processes correctly, $E_{\ell n}^{W}$ and $\Gamma_{\ell n}^{W}$ magnitudes satisfy Heisenberg principle for the whole range of thicknesses $(0 \leqslant \Delta \leqslant \infty)$.

Really, at the figure 5 there are presented the results of calculations for $E_{\ell n}^{W, S}$ and $\Gamma_{\ell n}^{W, S}$ dependence on $\Delta$ at $\ell=0,1,2, n=1,2,3$. Figure proves that for $\Delta \geqslant a_{0}$ the magnitudes: $E_{\ell n}^{W}, E_{\ell n}^{S}$ and $\Gamma_{\ell n}^{W}, \Gamma_{\ell n}^{S}$ coincide better at the increasing thickness. Herein, the accuracy of the resonance energies $\left(E_{\ell n}^{S}\right)$ is much better than that of resonance widths $\left(\Gamma_{\ell n}^{S}\right)$ for the same states $(\ell n)$.

For small thicknesses $\left(\Delta \leqslant a_{0}\right)$, the discrepancy between $E_{\ell n}^{W}$ and $E_{\ell n}^{S}$ as well as $\Gamma_{\ell n}^{W}$ and $\Gamma_{\ell n}^{S}$ 

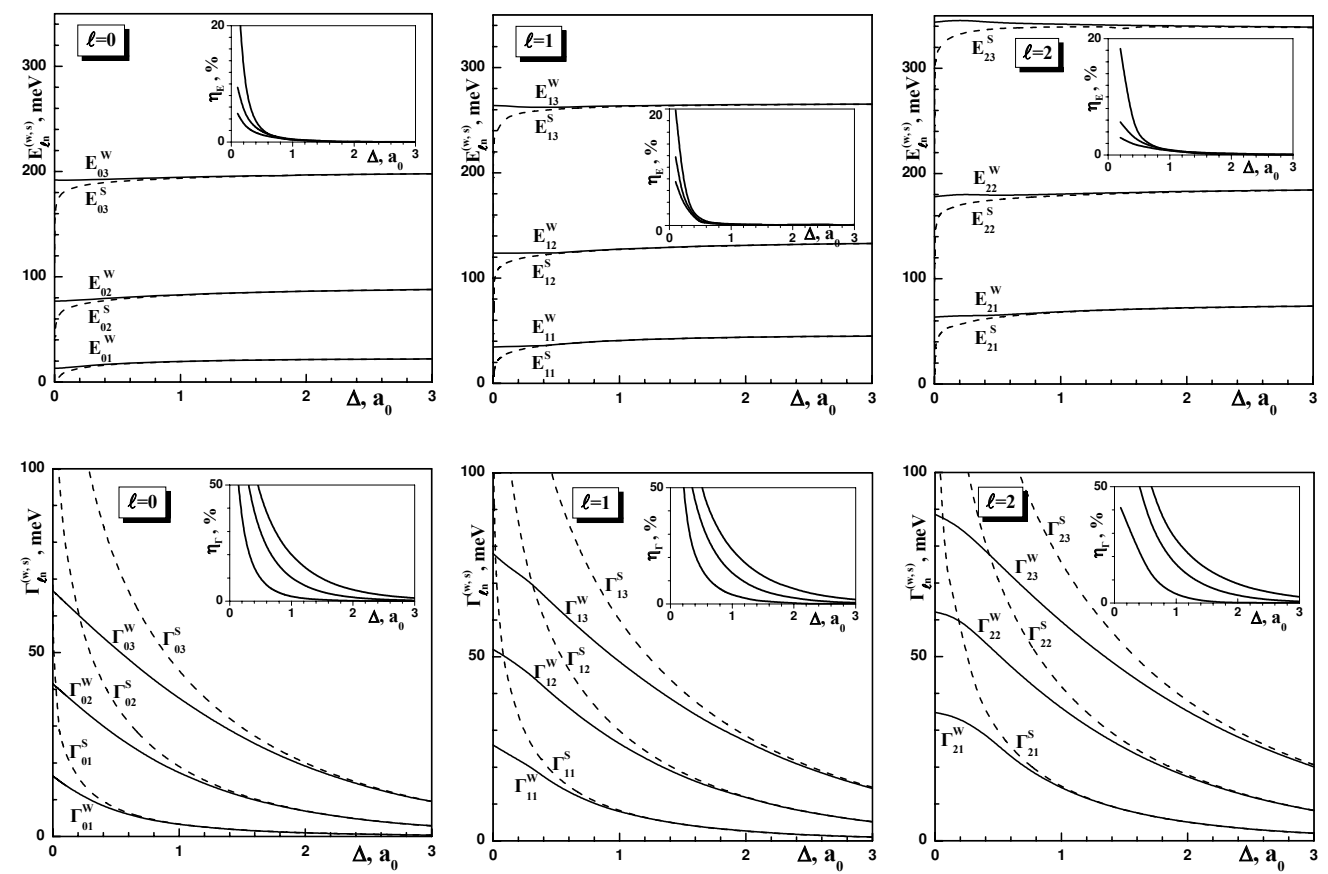

Figure 5. Dependences of $E_{\ell n}^{W, S}$ and $\Gamma_{\ell n}^{W, S}$ on barrier thickness $\Delta$ at $\ell=0,1,2, n=1,2,3$, $r_{0}=30 a_{0}$.

are rapidly increasing for the smaller $\Delta$. The error $\left(\eta_{\mathrm{E}}\right)$ of $E_{\ell n}^{S}$ and $\left(\eta_{\Gamma}\right) \Gamma_{\ell n}^{S}$ magnitudes, obtained within the $S_{\ell}$-matrix poles at a fixed thickness are increasing for the bigger orbital quantum number $(\ell)$ and number of resonance state $(n)$. Herein, the error $\eta_{\Gamma}$ of $\Gamma_{\ell n}^{S}$ magnitudes is much bigger than that $\eta_{\mathrm{E}}$ of $E_{\ell n}^{S}$. Already at $\Delta \approx 0.1 a_{0}$ one has $E_{\ell n}^{S} / \Gamma_{\ell n}^{S}=0.45<1 / 2$, contradicting Heisenberg principle of indeterminacy. Thus, the $S$-matrix method cannot be used for $E_{\ell n}^{S}$ and $\Gamma_{\ell n}^{S}$ calculations for small thicknesses $\Delta$. As far as the generalized resonance energies $\left(E_{\ell n}^{W}\right)$ and widths $\left(\Gamma_{\ell n}^{W}\right)$ are concerned, they perfectly satisfy Heisenberg principle of indeterminacy even at $\Delta=0$, as one can see in table 1.

Table 1. Dependence of $E_{\ell n}^{w} / \Gamma_{\ell n}^{w}$ on quantum numbers $n, \ell$ at $\Delta=0$.

\begin{tabular}{|l|c|c|c|}
\hline & $\mathbf{n = 1}$ & $\mathbf{n = 2}$ & $\mathbf{n}=\mathbf{3}$ \\
\hline$\ell=\mathbf{0}$ & 1.6 & 3.7 & 5.8 \\
\hline$\ell=\mathbf{1}$ & 2.7 & 4.7 & 6.8 \\
\hline$\ell=\mathbf{2}$ & 3.6 & 5.7 & 7.6 \\
\hline
\end{tabular}

\section{Conclusions}

1. The resonance energies $\left(E_{\ell n}^{S}\right)$ and widths $\left(\Gamma_{\ell n}^{S}\right)$ obtained as the poles of $S_{\ell-\text { matrix in complex }}$ plane satisfactorily characterize the electron quasistationary states in OSQD with the thickness of potential barrier bigger than the tenth part of the core radius. For smaller thicknesses, these spectral parameters loose their physical sense.

2. The probability distribution function $\left(W_{\ell}(E)\right)$ of electron location in QD and its parameters: generalized resonance energies $\left(E_{\ell n}^{W}\right)$ and widths $\left(\Gamma_{\ell n}^{W}\right)$ adequately describe the evolution of quasistationary states for the potential barrier thickness varying from zero up to infinity. 


\title{
References
}

1. Alferov J.I., Fiz. Tekh. Poluprovodn., 1998, 32, 3.

2. Ledentsov N.N., Ustinov V.M., Schukin V.A., Kopi'ev P.S., Alferov J.I., Bimberg D., Fiz. Tekh. Poluprovodn., 1998, 32, 385.

3. Schooss D., Mews A., Eychmuller A., Weller H., Phys. Rev. B, 1994, 49, 17072.

4. Chan W.C.W., Nie S., Science, 1998, 281, 2016.

5. Michalet X., Pihaud F.F., Bentolila L.A., Tsay J.M., Doose S., Li J.J., Sundaresan G., Wu A.M., Gambhir S.S., Weiss S., Science, 2005, 307, 538.

6. Zegrya G., Pis'ma Zh. Tekh. Fiz., 2006, 32, 75.

7. Tkach M., Holovatsky V., Fiz. Tverd. Tela, 1999, 41, 2081.

8. Tkach M., Holovatsky V., Fiz. Tverd. Tela,, 2001, 43, 350.

9. Tkach M., Holovatsky V., Voitsekhivska O., Physica E, 2001, 11, 17.

10. Tkach M., Makhanets A., Fiz. Tverd. Tela, 2005, 47, 550.

11. Tkach M., Seti Ju., Fiz. Tekh. Poluprovodn., 2006, 40, 1111.

12. Tkach M., Seti Ju., Zegrya G., Pis'ma Zh. Tekh. Fiz., 2007, 33, 70.

13. Baz' A., Zel'dovish Ia., Perelomov A. Scattering, reactions and decay in non relativistic quantum mechanics. Nauka, Moscow, 1971, 463.

\section{Еволюція квазістаціонарного спектра електрона у відкритій сферичній квантовій точці}

\author{
М.В.Ткач, Ю.О.Сеті, О.М.Войцехівська \\ Чернівецький національний університет ім. Юрія Федьковича, Чернівці 58012, вул. Коцюбинського 2 \\ Отримано 27 лютого 2008 р.

\begin{abstract}
Розглядається модель однієї ефективної маси та прямокутного потенціала. Методом S-матриці отримано точний розв'язок рівняння Шредінгера у загальному аналітичному вигляді. Вперше показано, що узагальнені резонансні енергії та ширини введені як параметри, що характеризують функцію розподілу (за енергією) ймовірності знаходження електрона в квантовій точці, адекватно (на відміну від полюсів S-матриці) характеризують еволюцію квазістаціонарних станів електрона на всьому інтервалі зміни товщини бар'єра від нуля (вільні стани) і до безмежності (стаціонарні зв'язані стани під бар'єром і віртуальні та вільні стани над бар'єром).
\end{abstract} \\ Вивчається еволюція квазістаціонарного спектру електрона у відкритій сферичній квантовій точці.
}

Ключові слова: сферична квантова точка, квазістаціонарний стан

PACS: 71.15.Dx, 73.21.La, 73.22.f, 73.90.+f 